\title{
Algebraic degrees of stretch factors in mapping class groups
}

\author{
HYUNSHIK SHIN
}

\begin{abstract}
We explicitly construct pseudo-Anosov maps on the closed surface of genus $g$ with orientable foliations whose stretch factor $\lambda$ is a Salem number with algebraic degree $2 g$. Using this result, we show that there is a pseudo-Anosov map whose stretch factor has algebraic degree $d$, for each positive even integer $d$ such that $d \leq g$.
\end{abstract}

57M50, 57M15

\section{Introduction}

Let $S_{g}$ be a closed surface of genus $g \geq 2$. The mapping class group of $S_{g}$, denoted $\operatorname{Mod}\left(S_{g}\right)$, is the group of isotopy classes of orientation-preserving homeomorphisms of $S_{g}$. An element $f \in \operatorname{Mod}\left(S_{g}\right)$ is called a pseudo-Anosov mapping class if there are transverse measured foliations $\left(\mathcal{F}^{u}, \mu_{u}\right)$ and $\left(\mathcal{F}^{s}, \mu_{s}\right)$, a number $\lambda(f)>1$, and a representative homeomorphism $\phi$ such that

$$
\phi\left(\mathcal{F}^{u}, \mu_{u}\right)=\left(\mathcal{F}^{u}, \lambda(f) \mu_{u}\right) \quad \text { and } \quad \phi\left(\mathcal{F}^{s}, \mu_{s}\right)=\left(\mathcal{F}^{s}, \lambda(f)^{-1} \mu_{s}\right) .
$$

In other words, $\phi$ stretches along one foliation by $\lambda(f)$ and the other by $\lambda(f)^{-1}$. The number $\lambda(f)$ is called the stretch factor (or dilatation) of $f$.

A pseudo-Anosov mapping class is said to be orientable if its invariant foliations are orientable. Let $\lambda_{H}(f)$ be the spectral radius of the action of $f$ on $H_{1}\left(S_{g} ; \mathbb{R}\right)$. Then

$$
\lambda_{H}(f) \leq \lambda(f),
$$

and the equality holds if and only if the invariant foliations for $f$ are orientable (see Lanneau and Thiffeault [5]). The number $\lambda_{H}(f)$ is called the homological stretch factor of $f$.

Question Which real numbers can be stretch factors?

This is a long-standing open question. Fried [4] conjectured that $\lambda>1$ is a stretch factor if and only if all conjugate roots of $\lambda$ and $1 / \lambda$ are strictly greater than $1 / \lambda$ and strictly less than $\lambda$ in magnitude. 
Thurston [12] showed that a stretch factor $\lambda$ is an algebraic integer whose algebraic degree has an upper bound $6 g-6$. More specifically, $\lambda$ is the largest root in absolute value of a monic palindromic polynomial. Thurston gave a construction of mapping classes of $\operatorname{Mod}\left(S_{g}\right)$ generated by two multitwists, and he mentioned that his construction can make a pseudo-Anosov mapping class whose stretch factor has algebraic degree $6 g-6$. However, he did not give specific examples.

What happens if we fix the genus $g$ ? To simplify the question, we may ask which algebraic degrees are possible on $S_{g}$.

Question What degrees of stretch factors can occur on $S_{g}$ ?

Very little is known about this question. Using Thurston's construction, it is easy to find quadratic integers as stretch factors. Neuwirth and Patterson [10] found nonquadratic examples, which are algebraic integers of degree 4 and 6 on surfaces of genus 4 and 6, respectively. Using interval exchange maps, Arnoux and Yoccoz [1] gave the first generic construction of pseudo-Anosov maps whose stretch factor has algebraic degree $g$ on $S_{g}$ for each $g \geq 2$.

\section{Main theorems}

In this paper, we give a generic construction of pseudo-Anosov mapping classes with stretch factor of algebraic degree $2 g$.

Let $c_{i}$ and $d_{j}$ be simple closed curves on $S_{g}$ as in Figure 1 . For $k \geq 3$, let us define

$$
f_{g, k}=T_{A_{g, k}} T_{B_{g}},
$$

where $T_{A_{g, k}}=\left(T_{c_{1}} T_{c_{2}} \cdots T_{c_{g-1}}\right)\left(T_{c_{g}}\right)^{k}$ and $T_{B_{g}}=T_{d_{1}} \cdots T_{d_{g}}$. Here, $T_{\alpha}$ is the Dehn twist about $\alpha$. We will show that $f_{g, k}$ is a pseudo-Anosov mapping class and its stretch factor $\lambda\left(f_{g, k}\right)$ is a special algebraic integer, called a Salem number. A Salem number is an algebraic integer $\alpha>1$ whose Galois conjugates other than $\alpha$ have absolute value less than or equal to 1 , and at least one of which lies on the unit circle.

Theorem A For each $g \geq 2$ and $k \geq 3, f_{g, k}$ is a pseudo-Anosov mapping class and satisfies the following properties:

(1) $\lambda\left(f_{g, k}\right)=\lambda_{H}\left(f_{g, k}\right)$,

(2) $\lambda\left(f_{g, k}\right)$ is a Salem number, and

(3) $\lim _{g \rightarrow \infty} \lambda\left(f_{g, k}\right)=k-1$.

In particular, we will prove that for $k=4$, the algebraic degree of the stretch factor is $2 g$. It is known that the degree of the stretch factor of a pseudo-Anosov mapping 


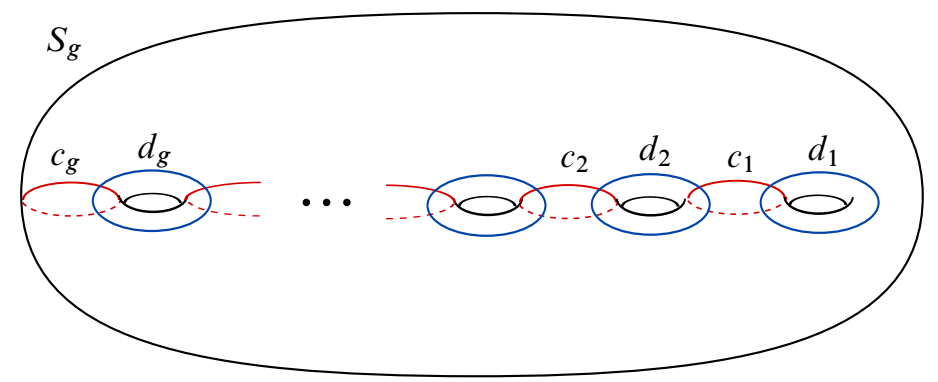

Figure 1: Simple closed curves on $S_{g}$

class $f \in \operatorname{Mod}\left(S_{g}\right)$ with orientable foliations is bounded above by $2 g$ (see [12]). Therefore our examples give the maximum degrees of stretch factors for orientable foliations in $\operatorname{Mod}\left(S_{g}\right)$ for each $g \geq 2$.

Theorem B Let $f_{g} \in \operatorname{Mod}\left(S_{g}\right)$ be the mapping class given by

$$
f_{g}=f_{g, 4}=T_{A_{g, 4}} T_{B_{g}}
$$

Then the minimal polynomial of the stretch factor $\lambda\left(f_{g}\right)$ is

This implies

$$
p_{g}(x)=x^{2 g}-2\left(\sum_{j=1}^{2 g-1} x^{j}\right)+1 .
$$

$$
\operatorname{deg} \lambda\left(f_{g}\right)=2 g
$$

The hard part is to show the irreducibility of $p_{g}(x)$, which is proved in Section 7.

In general, for each $k \geq 3$, the Salem stretch factor of $f_{g, k}$ is the root of the polynomial

$$
p_{g, k}(x)=x^{2 g}-(k-2)\left(\sum_{j=1}^{2 g-1} x^{j}\right)+1 .
$$

It can be shown that $p_{g, k}(x)$ is irreducible for each $k \geq 4$, but since the main purpose of this paper is degree realization, we will prove only for the $k=4$ case that the algebraic degree of the stretch factor is $2 g$.

Using a branched cover construction, we use Theorem B to deduce the following partial answer to our question about algebraic degrees.

Corollary 5 For each positive integer $h \leq g / 2$, there is a pseudo-Anosov mapping class $\tilde{f}_{h} \in \operatorname{Mod}\left(S_{g}\right)$ such that $\operatorname{deg}\left(\lambda\left(\tilde{f}_{h}\right)\right)=2 h$ and $\lambda\left(\tilde{f}_{h}\right)$ is a Salem number. 


\section{Obstructions}

There are three known obstructions for the existence of algebraic degrees. For any pseudo-Anosov $f \in \operatorname{Mod}\left(S_{g}\right)$, we have:

(1) $\operatorname{deg} \lambda(f) \geq 2$,

(2) $\operatorname{deg} \lambda(f) \leq 6 g-6$, and

(3) if $\operatorname{deg} \lambda(f)>3 g-3$, then $\operatorname{deg} \lambda(f)$ is even.

The third obstruction is due to Long [8] and we have another proof in Section 5. It turns out these are the only obstructions for $g=2$. However it is not known whether there are other obstructions of algebraic degrees for $g \geq 3$. By computer search, odd degree stretch factors are rare compared to even degrees. We conjecture that every even degree $d \leq 6 g-6$ can be realized as the algebraic degree of stretch factors.

Conjecture On $S_{g}$, there exists a pseudo-Anosov mapping class with a stretch factor of algebraic degree $d$ for each positive even integer $d \leq 6 g-6$.

In Section 6, we show that the conjecture is true for $g=2,3,4$ and 5 .

Outline In Section 2 we will give the basic definitions and results about Thurston's construction. We will prove Theorem A in Section 3 by the theory of Coxeter graphs. In Section 4, we construct pseudo-Anosov mapping classes via branched covers. In Section 5, we explain some properties of odd degree stretch factors. Section 6 contains examples of even degree stretch factors for $g=2,3,4$ and 5. Section 7 is where we prove Theorem $\mathrm{B}$, that is, we prove that the minimal polynomial of $\lambda\left(f_{g}\right)$ has degree $2 g$.

Acknowledgements I am very grateful to my advisor Dan Margalit for his help and numerous discussions. I would also like to thank Joan Birman, Benson Farb, Daniel Groves, Chris Judge, and Balázs Strenner for helpful suggestions and comments. I wish to thank an anonymous referee for very helpful comments. Lastly, I would like to thank the School of Mathematics of Georgia Institute of Technology for their hospitality during the time in which the major part of this paper was written.

This work was partially supported by the National Research Foundation of Korea (NRF) grant funded by the Korean government (MSIP) (No. 2016R1C1B1006843).

\section{Background}

\section{Thurston's construction}

We recall Thurston's construction of mapping classes [12]. For more details on this material, see [3] or [6]. 
Suppose $A=\left\{a_{1}, \ldots, a_{n}\right\}$ is a set of pairwise disjoint simple closed curves, called a multicurve. We denote the product of Dehn twists $\prod_{i=1}^{n} T_{a_{i}}$ by $T_{A}$. This product is called a multitwist.

Suppose $A=\left\{a_{1}, \ldots, a_{n}\right\}$ and $B=\left\{b_{1}, \ldots, b_{m}\right\}$ are multicurves in a surface $S$ so that $A \cup B$ fills $S$, that is, the complement of $A \cup B$ is a disjoint union of disks and once-punctured disks. Let $N$ be the $n \times m$ matrix whose $(j, k)$-entry is the geometric intersection number $i\left(a_{j}, b_{k}\right)$ of $a_{j}$ and $b_{k}$. Let $v=v(A \cup B)$ be the largest eigenvalue in magnitude of the matrix $N N^{t}$. If $A \cup B$ is connected, then $N N^{t}$ is primitive and by the Perron-Frobenius theorem $v$ is a positive real number greater than 1 (see [3, pages 392 - 395] for more detail).

Thurston constructed a singular Euclidean structure on $S$ with respect to which $\left\langle T_{A}, T_{B}\right\rangle$ acts by affine transformations given by the representation $\rho:\left\langle T_{A}, T_{B}\right\rangle \rightarrow$ $\operatorname{PSL}(2, \mathbb{R})$,

$$
\rho\left(T_{A}\right)=\left(\begin{array}{cc}
1 & -v^{1 / 2} \\
0 & 1
\end{array}\right) \text { and } \rho\left(T_{B}\right)=\left(\begin{array}{cc}
1 & 0 \\
v^{1 / 2} & 1
\end{array}\right)
$$

In particular, an element $f \in\left\langle T_{A}, T_{B}\right\rangle$ is pseudo-Anosov if and only if $\rho(f)$ is a hyperbolic element in $\operatorname{PSL}(2, \mathbb{R})$ and then the stretch factor $\lambda(f)$ is equal to the bigger eigenvalue of $\rho(f)$. For instance, for a mapping class $f=T_{A} T_{B}$,

$$
\rho\left(T_{A} T_{B}\right)=\left(\begin{array}{cc}
1 & -v^{1 / 2} \\
0 & 1
\end{array}\right)\left(\begin{array}{cc}
1 & 0 \\
v^{1 / 2} & 1
\end{array}\right)=\left(\begin{array}{cc}
1-v & -v^{1 / 2} \\
v^{1 / 2} & 1
\end{array}\right)
$$

and the stretch factor $\lambda\left(T_{A} T_{B}\right)$ is the bigger root of the characteristic polynomial

$$
\lambda^{2}-\lambda(v-2)+1,
$$

provided that $v-2>2$.

\section{Proof by the theory of Coxeter graphs}

We will prove Theorem A in this section.

For the set $C$ of simple closed curves on the surface $S_{g}$, the configuration graph for $C$, denoted $\mathcal{G}(C)$, is the graph with a vertex for each simple closed curve and an edge for every point of intersection between simple closed curves.

Let $f_{g, k}$ be a mapping class on $S_{g}$ defined by

$$
f_{g, k}=T_{A_{g, k}} T_{B_{g}}, \quad k \geq 3,
$$


as in Theorem A. By regarding the multiple power of $T_{c_{g}}$ as the product of Dehn twists about parallel (isotopic) simple closed curves $c_{g_{1}}, \ldots, c_{g_{k}}$, let us define the multicurves

$$
A_{g, k}=\left\{c_{1}, \ldots, c_{g-1}, c_{g_{1}}, \ldots, c_{g_{k}}\right\} \quad \text { and } \quad B_{g}=\left\{d_{1}, \ldots, d_{g}\right\} .
$$

Then the configuration graph $\mathcal{G}\left(A_{g, k} \cup B_{g}\right)$ is a tree as in Figure 2 .

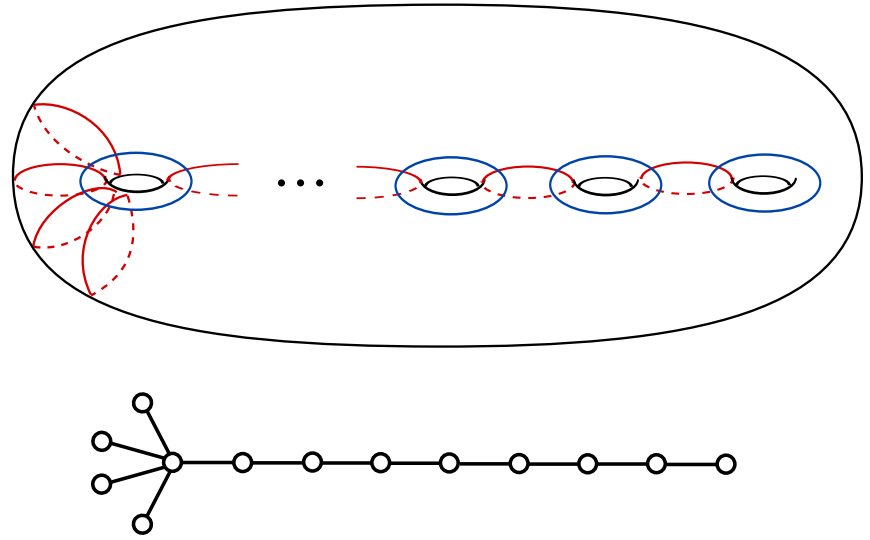

Figure 2: Multicurves and configuration graph $\mathcal{G}\left(A_{g, k} \cup B_{g}\right)$

\subsection{Coxeter graphs and mapping class groups}

We say that a finite graph $\mathcal{G}$ is a Coxeter graph if there are no self-loops or multiple edges. For given multicurves $A$ and $B$ such that $A \cup B$ fills the surface $S$, suppose that the configuration graph $\mathcal{G}=\mathcal{G}(A \cup B)$ is a Coxeter graph. Leininger proved the following theorem.

Theorem 1 [6, Theorem 8.1 and Theorem 8.4] Let $\mathcal{G}(A \cup B)$ be a noncritical dominant Coxeter graph. Then $T_{A} T_{B}$ is a pseudo-Anosov mapping class with stretch factor $\lambda$ such that

$$
\lambda^{2}+\lambda\left(2-\mu^{2}\right)+1=0,
$$

where $\mu$ is the spectral radius of the graph $\mathcal{G}$.

For the definitions and pictures of critical and dominant graphs, see [6, Section 1]

For the multicurves $A_{g, k}$ and $B_{g}$ in Theorem A, $\mathcal{G}\left(A_{g, k} \cup B_{g}\right)$ is a noncritical dominant Coxeter graph for each $k \geq 3$. Therefore by Theorem 1 the mapping class $f_{g, k}=T_{A} T_{B}$ is pseudo-Anosov for each $k \geq 3$. 


\subsection{Orientability}

Suppose that $\mathcal{G}$ is a connected Coxeter graph with the set $\Sigma$ of vertices. There is an associated quadratic form $\Pi_{\mathcal{G}}$ on $R^{\Sigma}$ and a faithful representation,

$$
\Theta: \mathscr{C}(\mathcal{G}) \rightarrow \mathrm{O}\left(\Pi_{\mathcal{G}}\right)
$$

where $\mathscr{C}(\mathcal{G})$ is a Coxeter group with generating set $\Sigma, \mathrm{O}\left(\Pi_{\mathcal{G}}\right)$ is the orthogonal group of the quadratic form $\Pi_{\mathcal{G}}$, and each generator $s_{i} \in \Sigma$ is represented by a reflection. Leininger also proved the following theorem.

Theorem 2 [6, Theorem 8.2] Let $\mathcal{G}(A \cup B)$ be a Coxeter graph and suppose that $A$ and $B$ can be oriented so that all intersections of $A$ with $B$ are positive. Then there exists a homomorphism

$$
\eta: \mathbb{R}^{\Sigma} \rightarrow H_{1}(S ; \mathbb{R})
$$

such that

$$
\left(T_{A} T_{B}\right)_{*} \circ \eta=-\eta \circ \Theta\left(\sigma_{A} \sigma_{B}\right),
$$

where $\sigma_{A} \sigma_{B}$ is an element in $\mathscr{C}(\mathcal{G})$ corresponding to $T_{A} T_{B}$. Moreover, we have $\left.\Theta\left(\sigma_{A} \sigma_{B}\right)\right|_{\operatorname{ker}(\eta)}=-I$ and $\eta$ preserves spectral radii.

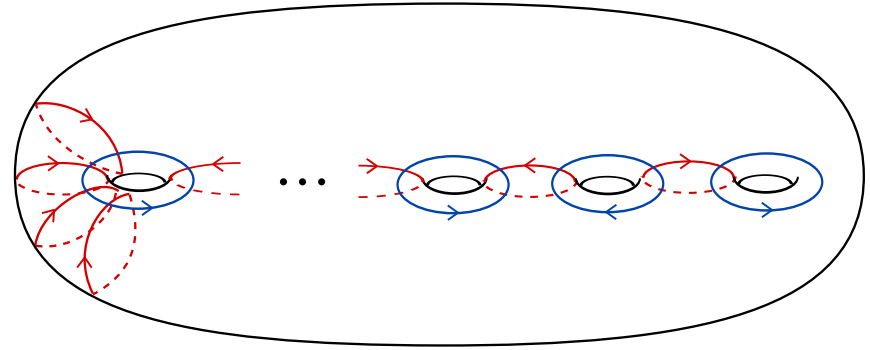

Figure 3: Orientation of positive intersections

Theorem 2 implies that if $A$ and $B$ can be oriented as described, then the stretch factor of a pseudo-Anosov mapping class is equal to the spectral radius of the action on homology. For multicurves $A_{g, k}$ and $B_{g}$ in Theorem A, they can be oriented so that all intersections are positive as in Figure 3. Therefore we have

$$
\lambda\left(f_{g, k}\right)=\lambda_{H}\left(f_{g, k}\right),
$$

and the invariant foliations for $f_{g, k}$ are orientable.

It is also possible to directly compute the action on the first homology. Consider the mapping class $f_{g}=T_{A_{g, 4}} T_{B_{g}}$ as in Theorem B. Let us choose a basis $\left\{a_{1}, b_{1}, \ldots, a_{g}, b_{g}\right\}$ for $H_{1}\left(S_{g}\right)$ as in Figure 4. 


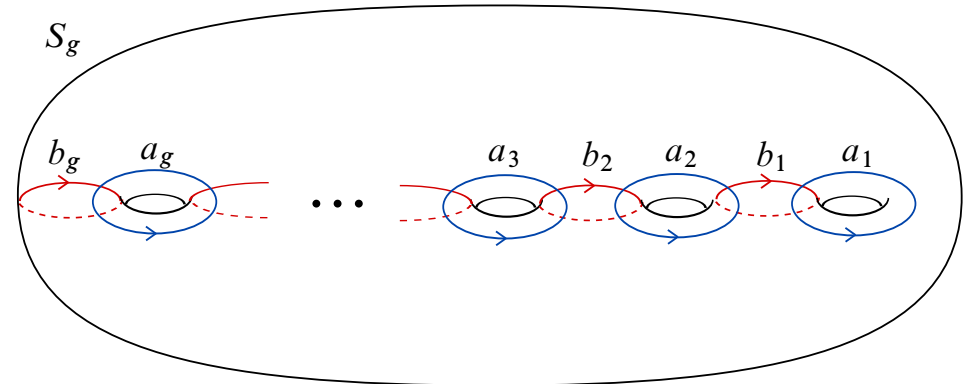

Figure 4: A basis for $H_{1}\left(S_{g}\right)$

By computing images of each basis element under $f_{g}$, we can get the action on $H_{1}\left(S_{g}\right)$ :

$$
\left(\begin{array}{rrrrrr}
1 & -1 & 0 & 0 & \cdots & 0 \\
1 & 0 & -1 & 0 & \cdots & 0 \\
\vdots & \vdots & \vdots & \vdots & \ddots & \vdots \\
1 & 0 & 0 & 0 & \cdots & -1 \\
4 & 0 & 0 & 0 & \cdots & -3
\end{array}\right) .
$$

By induction, the characteristic polynomial $h_{g}(x)$ of the homological action is

$$
h_{g}(x)=x^{2 g}+2\left(\sum_{j=1}^{2 g-1}(-1)^{j} x^{j}\right)+1 .
$$

Since the largest root of $h_{g}(x)$ in magnitude is a negative real number, we can deduce that the stretch factor $\lambda\left(f_{g}\right)$ is the root of $h_{g}(-x)$. Specifically, $\lambda\left(f_{g}\right)$ is the root of

$$
p_{g}(x)=x^{2 g}-2\left(\sum_{j=1}^{2 g-1} x^{j}\right)+1 .
$$

In a similar way, one can get the polynomial for $\lambda\left(f_{g, k}\right)$, which is

$$
p_{g, k}(x)=x^{2 g}-(k-2)\left(\sum_{j=1}^{2 g-1} x^{j}\right)+1 .
$$

\subsection{Salem numbers and spectral properties of starlike trees}

The configuration graph $\mathcal{G}\left(A_{g, k} \cup B_{g}\right)$ for $f_{g, k}$ is a special type of graph, called a starlike tree, and its relation to Salem numbers is studied in [9]. A starlike tree is a tree with at most one vertex of degree $>2$. Let $T=T\left(n_{1}, n_{2}, \ldots, n_{k}\right)$ be the starlike tree with $k$ arms of $n_{1}, n_{2}, \ldots, n_{k}$ edges. 
Theorem 3 [9, Corollary 9] Let $T=T\left(n_{1}, n_{2}, \ldots, n_{k}\right)$ be a starlike tree and let $\mu$ be the spectral radius of $T$. Suppose that $\mu$ is not an integer and $T$ is a noncritical dominant graph. Then $\lambda>1$, defined by $\sqrt{\lambda}+1 / \sqrt{\lambda}=\mu$, is a Salem number.

The configuration graph $\mathcal{G}\left(A_{g, k} \cup B_{g}\right)$ in Theorem A is a noncritical dominant starlike tree

$$
T(2 g-2, \underbrace{1,1, \ldots, 1}_{k \text { times }}), \quad k \geq 3
$$

and we will denote it by $T(2 g-2, k \cdot 1)$. The fact that the spectral radius of $T(2 g-2, k \cdot 1)$ is not an integer follows from the following theorem.

Theorem 4 [11] If $\mu$ is the spectral radius of the starlike tree $T(n, k \cdot 1)$, then

$$
\sqrt{k+1}<\mu<\frac{k}{\sqrt{k-1}}
$$

for $n \geq 1$ and $k \geq 3$.

Thus for the starlike tree $T(n, k \cdot 1)$, the spectral radius satisfies

$$
k+1<\mu^{2}<\frac{k^{2}}{k-1}=k+1+\frac{1}{k-1} .
$$

Therefore $\mu$ is not an integer and by Theorem $3, \lambda\left(f_{g, k}\right)$ is a Salem number.

Moreover, the proof of Lepović and Gutman [7, Corollary 2.1] implies that

$$
\lim _{g \rightarrow \infty} \lambda\left(f_{g, k}\right)=k-1 .
$$

For completeness, we reprove this here.

Recall that $\lambda\left(f_{g, k}\right)$ is the largest root of

$$
p_{g, k}(x)=x^{2 g}-(k-2)\left(\sum_{j=1}^{2 g-1} x^{j}\right)+1 .
$$

By multiplying $p_{g, k}(x)$ by $x-1$, the stretch factor $\lambda\left(f_{g, k}\right)$ is the largest root in magnitude of

$$
q_{g, k}(x)=x^{2 g+1}-(k-1) x^{2 g}+(k-1) x-1 .
$$

We have $q_{g, k}(k-1)=(k-1)^{2}-1>0$, and for any fixed positive integer $m$,

$$
q_{g, k}\left(k-1-\frac{1}{10^{m}}\right)=\left(k-1-\frac{1}{10^{m}}\right)^{2 g}\left(-\frac{1}{10^{m}}\right)+(k-1)\left(k-1-\frac{1}{10^{m}}\right)-1 .
$$


Hence $q_{g, k}\left(k-1-10^{-m}\right)<0$ for sufficiently large values of $g$ and therefore $p_{g, k}(x)$ has a root on the interval $\left(k-1-10^{-m}, k-1\right)$. This implies

$$
\lim _{g \rightarrow \infty} \lambda\left(f_{g, k}\right)=k-1 \text {. }
$$

This completes the proof of Theorem A.

Remark A positive integer cannot be a stretch factor (which is an algebraic integer of degree 1). However, Theorem A implies that for sufficiently large genus $g$ there is a stretch factor which is a Salem number arbitrarily close to a given integer $k-1$ for each $k \geq 3$.

\section{Branched covers}

Lifting a pseudo-Anosov mapping class via a covering map is one way to construct another pseudo-Anosov mapping class. If there is a branched cover $\widetilde{S} \rightarrow S$ and a pseudoAnosov mapping class $f \in \operatorname{Mod}(S)$, then there is some $k \in \mathbb{N}$ such that $\operatorname{Mod}(\widetilde{S})$ has a pseudo-Anosov element $\tilde{f}$ which is a lift of $f^{k}$ and hence $\lambda(\tilde{f})=\lambda(f)^{k}$.

Corollary 5 Let $g \geq 2$. For each positive integer $h \leq g / 2$, there is a pseudo-Anosov mapping class $\tilde{f}_{h} \in \operatorname{Mod}\left(S_{g}\right)$ such that $\lambda\left(\tilde{f}_{h}\right)$ is a Salem number and $\operatorname{deg}\left(\lambda\left(\tilde{f}_{h}\right)\right)=2 h$.

Proof Let

$$
h= \begin{cases}\frac{1}{2}(g-2 m) & \text { if } g \text { is even, } m=0,1, \ldots,(g-2) / 2, \\ \frac{1}{2}(g-1-2 m) & \text { if } g \text { is odd, } m=0,1, \ldots,(g-3) / 2 .\end{cases}
$$

Then $h$ is an integer such that $1 \leq h \leq g / 2$.

Construct a branched cover $S_{g} \rightarrow S_{h}$ as in Figure 5. For $h \geq 2, S_{h}$ has a pseudoAnosov mapping class $f_{h} \in \operatorname{Mod}\left(S_{h}\right)$ as in Theorem B whose stretch factor has $\operatorname{deg}\left(\lambda\left(f_{h}\right)\right)=2 h$. For some $k, f_{h}{ }^{k}$ lifts to $S_{g}$ and the lift has stretch factor $\lambda\left(f_{h}\right)^{k}$. We claim that $\operatorname{deg}\left(\lambda\left(f_{h}\right)^{k}\right)=2 h$. To see this, let $\lambda_{i}, 1 \leq i \leq 2 h$, be the roots of the minimal polynomial of $\lambda\left(f_{h}\right)$ and let us define a polynomial

$$
p(x)=\prod_{i=1}^{2 h}\left(x-\lambda_{i}^{k}\right)
$$

Then $p(x)$ is an integral polynomial because the elementary symmetric polynomials

$$
\sum \lambda_{i}, \quad \sum_{i<j} \lambda_{i} \lambda_{j}, \quad \sum_{i<j<l} \lambda_{i} \lambda_{j} \lambda_{l}, \quad \ldots, \quad \lambda_{1} \lambda_{2} \cdots \lambda_{2 h}
$$



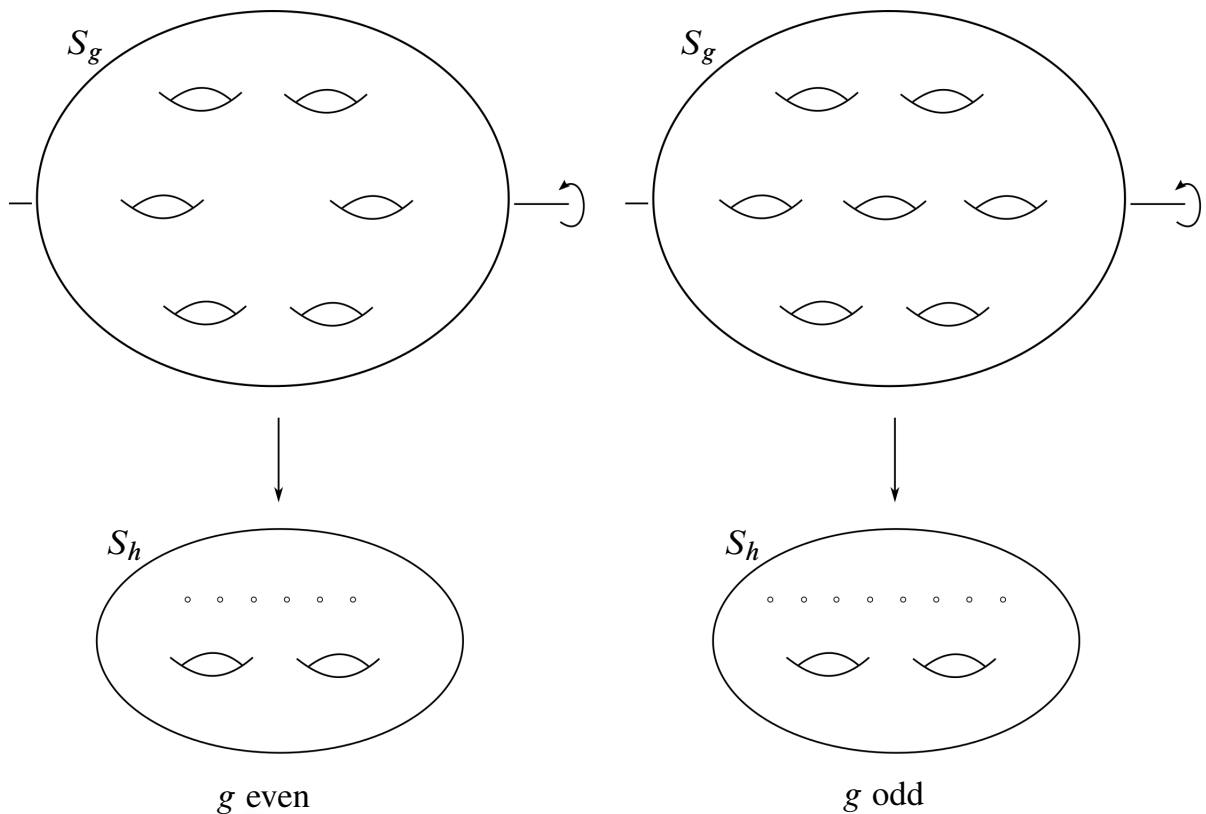

Figure 5: A branched cover

in $\lambda_{1}, \ldots, \lambda_{2 h}$ are all integers, and hence the coefficients

$$
\sum \lambda_{i}^{k}, \quad \sum_{i<j} \lambda_{i}^{k} \lambda_{j}^{k}, \quad \sum_{i<j<l} \lambda_{i}^{k} \lambda_{j}^{k} \lambda_{l}^{k}, \quad \ldots, \quad \lambda_{1}^{k} \lambda_{2}^{k} \cdots \lambda_{2 h}^{k}
$$

of $p(x)$ are integers as well. Therefore $p(x)$ is divided by the minimal polynomial of $\lambda\left(f_{h}\right)^{k}$. Due to the proof of Theorem B in Section $7, \lambda\left(f_{h}\right)^{k}$ is also a Salem number and $p(x)$ does not have a cyclotomic factor. This implies that $p(x)$ is irreducible and $\operatorname{deg}\left(\lambda\left(f_{h}\right)^{k}\right)=2 h$.

If $h=1, S_{h}$ is a torus and it admits an Anosov mapping class $f$ whose stretch factor $\lambda(f)$ has algebraic degree 2 . Then similar arguments to those above tell us that there is a lift of some power of $f$ to $S_{g}$ whose stretch factor has $\operatorname{deg}\left(\lambda\left(f^{k}\right)\right)=2$.

Therefore there is a pseudo-Anosov map $\tilde{f}_{h} \in \operatorname{Mod}\left(S_{g}\right)$ with $\operatorname{deg}\left(\lambda\left(\tilde{f}_{h}\right)\right)=2 h$ for each $h \leq g / 2$. In other words, every positive even degree $d \leq g$ is realized as the algebraic degree of a stretch factor on $S_{g}$.

\section{Stretch factors of odd degrees}

Long proved the following degree obstruction and McMullen communicated to us the following proof. First we will give a definition of the reciprocal polynomial. Given a 
polynomial $p(x)$ of degree $d$, we define the reciprocal polynomial $p^{*}(x)$ of $p(x)$ by $p^{*}(x)=x^{d} p(1 / x)$. It is a well-known property that $p^{*}(x)$ is irreducible if and only if $p(x)$ is irreducible.

Theorem 6 [8] Let $f \in \operatorname{Mod}\left(S_{g}\right)$ be a pseudo-Anosov mapping class having stretch factor $\lambda(f)$. If $\operatorname{deg}(\lambda(f))>3 g-3$, then $\operatorname{deg}(\lambda(f))$ is even.

Proof Since $f$ acts by a piecewise integral projective transformation on the $6 g-6$ dimensional space $\mathcal{P} \mathcal{M F}$ of projective measured foliations on $S_{g}$, and since $\lambda(f)$ is an eigenvalue of this action, $\lambda(f)$ is an algebraic integer with $\operatorname{deg}(\lambda(f)) \leq 6 g-6$. Also, since $f$ preserves the symplectic structure on $\mathcal{P} \mathcal{M F}$, it follows that $\lambda(f)$ is the root of the palindromic polynomial $p(x)$ whose degree is bounded above by $6 g-6$.

Let $q(x)$ be the minimal polynomial of $\lambda(f)$ and let $q^{*}(x)$ be the reciprocal polynomial of $q(x)$. Then either $q(x)=q^{*}(x)$ or they have no common roots, because if there is at least one common root $\zeta$ of $q(x)$ and $q^{*}(x)$, then both $q(x)$ and $q^{*}(x)$ are the minimal polynomial of $\zeta$ and hence $q(x)=q^{*}(x)$. Suppose $\operatorname{deg}(q(x))>3 g-3$. If $q(x)$ and $q^{*}(x)$ have no common roots, then their product $q(x) q^{*}(x)$ is a factor of $p(x)$ since $q^{*}(x)$ is the minimal polynomial of $1 / \lambda(f)$. This is a contradiction because $\operatorname{deg}(p(x)) \leq 6 g-6$ but $\operatorname{deg}\left(q(x) q^{*}(x)\right)>6 g-6$. Therefore we must have $q(x)=q^{*}(x)$ and this implies that $q(x)$ is an irreducible palindromic polynomial. Hence $\operatorname{deg}(q(x))$ is even since roots of $q(x)$ come in pairs, $\lambda_{i}$ and $1 / \lambda_{i}$.

It follows from the previous proof that if the minimal polynomial $p(x)$ of $\lambda$ has odd degree, then $p(x)$ is not palindromic and in fact the minimal palindromic polynomial containing $\lambda$ as a root is $p(x) p^{*}(x)$.

We will now show that the stretch factors of degree 3 have an additional special property. A Pisot number, also called a Pisot-Vijayaraghavan number or a PV number, is an algebraic integer greater than 1 such that all its Galois conjugates are strictly less than 1 in absolute value.

Proposition 7 Let $f \in \operatorname{Mod}\left(S_{g}\right)$. If $\operatorname{deg}(\lambda(f))=3$, then $\lambda(f)$ is a Pisot number.

Proof Let $\lambda_{1}>1$ be the stretch factor of a pseudo-Anosov mapping class with algebraic degree 3 , and let $p(x)$ be the minimal polynomial of $\lambda_{1}$. Let $\lambda_{1}, \lambda_{2}$, and $\lambda_{3}$ be the roots of $p(x)$. Then the degree of $p(x) p^{*}(x)$ is 6 and it has pairs of roots $\left(\lambda_{1}, 1 / \lambda_{1}\right),\left(\lambda_{2}, 1 / \lambda_{2}\right),\left(\lambda_{3}, 1 / \lambda_{3}\right)$, where $\lambda_{1}$ is the largest root in absolute value. We claim that the absolute values of $\lambda_{2}$ and $\lambda_{3}$ are strictly less than 1 . 
Suppose one of them has absolute value greater than or equal to 1 , say $\left|\lambda_{2}\right| \geq 1$. The constant term $\lambda_{1} \lambda_{2} \lambda_{3}$ of $p(x)$ is \pm 1 since it is the factor of a palindromic polynomial with constant term 1 . Hence $\left|\lambda_{1} \lambda_{2} \lambda_{3}\right|=1$ and we have

$$
\frac{1}{\left|\lambda_{3}\right|}=\left|\lambda_{1} \lambda_{2}\right| \geq\left|\lambda_{1}\right|
$$

which is a contradiction to the fact that the stretch factor $\lambda_{1}$ is strictly greater than all other roots of the palindromic polynomial $p(x) p^{*}(x)$. This proves the claim and hence the stretch factor of degree 3 is a Pisot number.

We now explain two constructions of mapping classes $f \in \operatorname{Mod}\left(S_{g}\right)$ whose degree of $\lambda(f)$ is odd.

(1) As we mentioned, Arnoux and Yoccoz [1] gave examples of a pseudo-Anosov mapping class on $S_{g}$ whose stretch factor has algebraic degree $g$. In particular, for odd $g$, this gives examples of mapping classes with odd degree stretch factors. They proved that these stretch factors are all Pisot numbers.

(2) For genus 2, there is a pseudo-Anosov mapping class $f$ whose stretch factor has algebraic degree 3 (see Section 6). This is the only possible odd degree on $S_{2}$ by Long's obstruction. It is also true that $\operatorname{deg}\left(\lambda(f)^{k}\right)=3$ for each $k$ because the stretch factor is a Pisot number (Proposition 7). There is a cover $S_{g} \rightarrow S_{2}$ for each $g$, so the lift of some power of $f$ has a stretch factor with algebraic degree 3 on $S_{g}$ :

Proposition 8 For each genus $g$, the stretch factor with algebraic degree 3 can occur on $S_{g}$.

Question Are there stretch factors with odd algebraic degree that are not Pisot numbers?

\section{Examples of even degrees}

Tables 1-4 give explicit examples of pseudo-Anosov mapping classes whose stretch factors realize various degrees. We will follow the notation of the software Xtrain by Brinkmann. More specifically, $a_{i}, b_{i}, c_{i}$ and $d_{i}$ are Dehn twists along standard curves and $A_{i}, B_{i}, C_{i}$ and $D_{i}$ are the inverse twists as in [2]. The only missing degree on $S_{3}$ is degree 5 . We do not know if there is a degree 5 example or there is another degree obstruction. 


\begin{tabular}{|clll|}
\hline deg & $f \in \operatorname{Mod}\left(S_{2}\right)$ & Minimal polynomial & $\lambda(f)$ \\
\hline 2 & $a_{0} a_{0} d_{0} C_{0} D_{1} C_{0}$ & $x^{2}-3 x+1$ & 2.618 \\
3 & $a_{0} d_{0} d_{0} C_{0} C_{0} D_{1}$ & $x^{3}-3 x^{2}-x-1$ & 3.383 \\
4 & $a_{0} d_{0} d_{0} d_{1} c_{0} d_{0}$ & $x^{4}-x^{3}-x^{2}-x+1$ & 1.722 \\
6 & $a_{0} a_{0} d_{0} A_{0} C_{0} D_{1}$ & $x^{6}-x^{5}-4 x^{3}-x+1$ & 2.015 \\
\hline
\end{tabular}

Table 1: Examples of genus 2

\begin{tabular}{|clll|}
\hline deg & $f \in \operatorname{Mod}\left(S_{3}\right)$ & Minimal polynomial & $\lambda(f)$ \\
\hline 2 & $a_{1} c_{0} d_{0} c_{0} d_{2} C_{1} D_{1}$ & $x^{2}-4 x+1$ & 3.732 \\
3 & $a_{0} c_{0} d_{0} C_{1} D_{1} D_{2}$ & $x^{3}-2 x^{2}+x-1$ & 1.755 \\
4 & $a_{1} c_{0} d_{0} a_{1} c_{1} d_{1} d_{2}$ & $x^{4}-x^{3}-2 x^{2}-x+1$ & 1.722 \\
6 & $a_{0} c_{0} d_{0} d_{2} C_{1} D_{1}$ & $x^{6}-3 x^{5}+3 x^{4}-7 x^{3}+3 x^{2}-3 x+1$ & 2.739 \\
8 & $a_{0} c_{0} d_{0} d_{1} C_{1} D_{2}$ & $x^{8}-x^{7}-2 x^{5}-2 x^{3}-x+1$ & 1.809 \\
10 & $a_{1} c_{0} d_{0} d_{1} C_{1} A_{2} D_{2}$ & $x^{10}-x^{9}-2 x^{8}+2 x^{7}-2 x^{5}+2 x^{3}-2 x^{2}-x+1$ & 1.697 \\
12 & $a_{1} c_{1} c_{0} d_{1} d_{2} A_{0} D_{0}$ & $x^{12}-x^{11}-x^{9}-x^{8}+x^{7}+x^{5}-x^{4}-x^{3}-x+1$ & 1.533 \\
\hline
\end{tabular}

Table 2: Examples of genus 3

\begin{tabular}{|cl|cl|}
\hline deg & $f \in \operatorname{Mod}\left(S_{4}\right)$ & $\operatorname{deg}$ & $f \in \operatorname{Mod}\left(S_{4}\right)$ \\
\hline 4 & $a_{0} a_{0} a_{1} c_{0} d_{0} c_{1} d_{1} c_{2} d_{2} c_{3} d_{3}$ & 12 & $a_{0} B_{1} d_{0} c_{0} d_{1} c_{1} d_{2} c_{2} d_{3} c_{3}$ \\
6 & $a_{0} B_{2} A_{3} d_{0} c_{0} d_{1} c_{1} d_{2} c_{2} d_{3} c_{3}$ & 14 & $a_{0} d_{0} B_{0} d_{0} c_{0} d_{1} c_{1} d_{2} c_{2} d_{3} c_{3}$ \\
8 & $a_{0} A_{1} d_{0} c_{0} d_{1} c_{1} d_{2} c_{2} d_{3} c_{3}$ & 16 & $A_{0} d_{0} c_{0} d_{1} c_{1} d_{2} c_{2} d_{3} c_{3}$ \\
10 & $a_{0} b_{1} A_{2} d_{0} c_{0} d_{1} c_{1} d_{2} c_{2} d_{3} c_{3}$ & 18 & $a_{0} B_{1} A_{2} d_{0} c_{0} d_{1} c_{1} d_{2} c_{2} d_{3} c_{3}$ \\
\hline
\end{tabular}

Table 3: Examples of genus 4

\begin{tabular}{|cl|cl|}
\hline $\operatorname{deg}$ & $f \in \operatorname{Mod}\left(S_{5}\right)$ & $\operatorname{deg}$ & $f \in \operatorname{Mod}\left(S_{5}\right)$ \\
\hline 6 & $b_{3} d_{0} c_{0} d_{1} c_{1} d_{2} c_{2} d_{3} c_{3} d_{4} c_{4}$ & 16 & $a_{1} B_{2} d_{0} c_{0} d_{1} c_{1} d_{2} c_{2} d_{3} c_{3} d_{4} c_{4}$ \\
8 & $a_{0} a_{1} d_{0} c_{0} d_{1} c_{1} d_{2} c_{2} d_{3} c_{3} d_{4} c_{4}$ & 18 & $a_{1} B_{0} d_{0} c_{0} d_{1} c_{1} d_{2} c_{2} d_{3} c_{3} d_{4} c_{4}$ \\
10 & $a_{1} A_{4} d_{0} c_{0} d_{1} c_{1} d_{2} c_{2} d_{3} c_{3} d_{4} c_{4}$ & 20 & $a_{1} A_{0} d_{0} c_{0} d_{1} c_{1} d_{2} c_{2} d_{3} c_{3} d_{4} c_{4}$ \\
12 & $b_{2} C_{2} d_{0} c_{0} d_{1} c_{1} d_{2} c_{2} d_{3} c_{3} d_{4} c_{4}$ & 22 & $a_{2} A_{1} d_{0} c_{0} d_{1} c_{1} d_{2} c_{2} d_{3} c_{3} d_{4} c_{4}$ \\
14 & $a_{1} B_{1} d_{0} c_{0} d_{1} c_{1} d_{2} c_{2} d_{3} c_{3} d_{4} c_{4}$ & 24 & $c_{2} A_{2} d_{0} c_{0} d_{1} c_{1} d_{2} c_{2} d_{3} c_{3} d_{4} c_{4}$ \\
\hline
\end{tabular}

Table 4: Examples of genus 5 


\section{Irreducibility of polynomials}

In this section, we will prove Theorem B. It is enough to show that the polynomial

$$
p_{n}(x)=x^{2 n}-2\left(\sum_{j=1}^{2 n-1} x^{j}\right)+1
$$

is irreducible for $n \geq 2$. We will show that $p_{n}(x)$ does not have a cyclotomic polynomial factor. It then follows from Kronecker's theorem that $p_{n}(x)$ is irreducible.

Suppose $p_{n}(x)$ has the $m^{\text {th }}$ cyclotomic polynomial factor for some $m \in \mathbb{N}$. Then $e^{2 \pi i / m}$ is a root of $p_{n}(x)$. Multiplying $p_{n}(x)$ by $x-1$ yields

$$
x^{2 n+1}-3 x^{2 n}+3 x-1,
$$

and hence we have

$$
e^{2(2 n+1) \pi i / m}-3 e^{4 n \pi i / m}+3 e^{2 \pi i / m}-1=0 .
$$

Consider the real part and the complex part of (1). Then we have the system of equations

$$
\left\{\begin{array}{l}
\cos \frac{2(2 n+1) \pi}{m}-3 \cos \frac{4 n \pi}{m}+3 \cos \frac{2 \pi}{m}-1=0, \\
\sin \frac{2(2 n+1) \pi}{m}-3 \sin \frac{4 n \pi}{m}+3 \sin \frac{2 \pi}{m}=0 .
\end{array}\right.
$$

Using the double-angle formula for the first cosine and sum-to-product formula for the last two cosines, the first equation gives

$$
2 \sin \left(\frac{(2 n+1) \pi}{m}\right)\left[3 \sin \frac{(2 n-1) \pi}{m}-\sin \frac{(2 n+1) \pi}{m}\right]=0
$$

Similarly, the second equation gives

$$
2 \cos \left(\frac{(2 n+1) \pi}{m}\right)\left[\sin \frac{(2 n+1) \pi}{m}-3 \sin \frac{(2 n-1) \pi}{m}\right]=0 .
$$

Since sine and cosine have no common zeros, we must have

$$
\sin \frac{(2 n+1) \pi}{m}-3 \sin \frac{(2 n-1) \pi}{m}=0 .
$$

For $m \leq 5$, by direct calculation we can see that $p_{n}\left(e^{2 \pi i / m}\right) \neq 0$. So we may assume that $m \geq 6$. Let $\varphi=(2 n-1) \pi / m$. Then we can write the above equation as

$$
\sin \left(\varphi+\frac{2 \pi}{m}\right)-3 \sin \varphi=0 .
$$


Since $\sin (\varphi+2 \pi / m)$ is a real number between -1 and 1 , we have

$$
-\frac{1}{3} \leq \sin \varphi \leq \frac{1}{3} \text {. }
$$

Let $\psi=\sin ^{-1}\left(\frac{1}{3}\right)$. Then note that $\psi<\pi / 6$. Equation (3) gives the restriction on $\varphi$, which is

$$
-\psi \leq \varphi \leq \psi \quad \text { or } \quad \pi-\psi \leq \varphi \leq \pi+\psi .
$$

Another observation from (2) is that both $\sin (\varphi+2 \pi / m)$ and $\sin \varphi$ must have the same sign.

We claim that $\varphi$ has to be in either the first or third quadrant. Suppose $\varphi$ is in the second quadrant, that is, $\pi-\psi<\varphi<\pi$. Note that $m \geq 6$ implies $2 \pi / m \leq \pi / 3$. Since $\varphi$ is above the $x$-axis, $\varphi+2 \pi / m$ also has to be above the $x$-axis due to (2) and hence the only possibility is that $\varphi+2 \pi / m$ is between $\varphi$ and $\pi$. Then

$$
0<\sin \left(\varphi+\frac{2 \pi}{m}\right)<\sin \varphi \Rightarrow \sin \left(\varphi+\frac{2 \pi}{m}\right)<3 \sin \varphi,
$$

which is a contradiction to (2). Similar arguments hold if $\varphi$ is in the fourth quadrant. Therefore the possible range for $\varphi$ is

$$
0<\varphi \leq \psi \quad \text { or } \quad \pi<\varphi \leq \pi+\psi .
$$

Suppose $\varphi$ is in the first quadrant. Then so is $\varphi+2 \pi / m$ because

$$
0<\varphi+\frac{2 \pi}{m} \leq \psi+\frac{\pi}{3}<\frac{\pi}{2} .
$$

We can write

$$
\varphi=\frac{(2 n-1) \pi}{m} \equiv \frac{j \pi}{m}(\bmod 2 \pi)
$$

for some positive integer $j$, ie, $0<j \pi / m<\pi / 2$.

If $j \geq 2$, using the subadditivity of $\sin x$ in the first quadrant,

$$
\sin (x+y) \leq \sin x+\sin y,
$$

we have

$$
\begin{aligned}
\sin \left(\varphi+\frac{2 \pi}{m}\right)-3 \sin \varphi & \leq\left(\sin \varphi+\sin \frac{2 \pi}{m}\right)-3 \sin \varphi \\
& =\sin \frac{2 \pi}{m}-2 \sin \varphi \\
& =\sin \frac{2 \pi}{m}-2 \sin \frac{j \pi}{m}<0,
\end{aligned}
$$

which contradicts (2). 
If $j=1$, using the triple-angle formula, we obtain

$$
\begin{aligned}
\sin \left(\varphi+\frac{2 \pi}{m}\right)-3 \sin \varphi & =\sin \frac{3 \pi}{m}-3 \sin \frac{\pi}{m} \\
& =\left(3 \sin \frac{\pi}{m}-4 \sin ^{3} \frac{\pi}{m}\right)-3 \sin \frac{\pi}{m} \\
& =-4 \sin ^{3} \frac{\pi}{m}<0,
\end{aligned}
$$

which contradicts (2) again. Therefore there is no possible $\varphi$ in the first quadrant. The same argument gives a contradiction if $\varphi$ is in the third quadrant. Therefore we can conclude that $p(x)$ does not have a cyclotomic factor.

We now show that $p_{n}(x)$ is irreducible over $\mathbb{Z}$. Suppose $p_{n}(x)$ is reducible and write $p_{n}(x)=g(x) h(x)$ with nonconstant functions $g(x)$ and $h(x)$. There is only one root of $p_{n}(x)$ whose absolute value is strictly greater than 1 . Therefore one of $g(x)$ or $h(x)$ has all roots inside the unit disk. By Kronecker's theorem, this polynomial has to be a product of cyclotomic polynomials, which is a contradiction because $p_{n}(x)$ does not have a cyclotomic polynomial factor. Therefore $p_{n}(x)$ is irreducible.

\section{References}

[1] P Arnoux, J-C Yoccoz, Construction de difféomorphismes pseudo-Anosov, C. R. Acad. Sci. Paris Sér. I Math. 292 (1981) 75-78 MR610152

[2] P Brinkmann, An implementation of the Bestvina-Handel algorithm for surface homeomorphisms, Experiment. Math. 9 (2000) 235-240 MR1780208

[3] B Farb, D Margalit, A primer on mapping class groups, Princeton Mathematical Series 49, Princeton Univ. Press (2012) MR2850125

[4] D Fried, Growth rate of surface homeomorphisms and flow equivalence, Ergodic Theory Dynam. Systems 5 (1985) 539-563 MR829857

[5] E Lanneau, J-L Thiffeault, On the minimum dilatation of pseudo-Anosov homeromorphisms [sic] on surfaces of small genus, Ann. Inst. Fourier (Grenoble) 61 (2011) 105-144 MR2828128

[6] C J Leininger, On groups generated by two positive multi-twists: Teichmüller curves and Lehmer's number, Geom. Topol. 8 (2004) 1301-1359 MR2119298

[7] M Lepović, I Gutman, Some spectral properties of starlike trees, Bull. Cl. Sci. Math. Nat. Sci. Math. 122 (2001) 107-113 MR1874622

[8] D D Long, Constructing pseudo-Anosov maps, from: "Knot theory and manifolds", (D Rolfsen, editor), Lecture Notes in Math. 1144, Springer, Berlin (1985) 108-114 MR823284 
[9] J F McKee, P Rowlinson, C J Smyth, Salem numbers and Pisot numbers from stars, from: "Number theory in progress, Vol. 1", (K Györy, H Iwaniec, J Urbanowicz, editors), de Gruyter, Berlin (1999) 309-319 MR1689512

[10] L Neuwirth, N Patterson, A sequence of pseudo-Anosov diffeomorphisms, from: "Combinatorial group theory and topology", (S M Gersten, S J R, editors), Ann. of Math. Stud. 111, Princeton Univ. Press (1987) 443-449 MR895627

[11] H Shin, Spectral radius of a star with one long arm, preprint (2015) To appear in Open J. Discrete Math. Available at http://mathsci.kaist.ac.kr/ hshin/papers/ starlikelongarm.pdf

[12] W P Thurston, On the geometry and dynamics of diffeomorphisms of surfaces, Bull. Amer. Math. Soc. 19 (1988) 417-431 MR956596

Department of Mathematical Sciences, KAIST

291 Daehak-ro, Yuseong-gu, Daejeon 34141, South Korea

hshin@kaist.ac.kr

http://mathsci.kaist.ac.kr/ hshin

Received: 25 September 2014 Revised: 16 September 2015 